\title{
Death NOS, CTCAE
}

National Cancer Institute

\section{Source}

National Cancer Institute. Death NOS, CT CAE. NCI Thesaurus. Code C143396.

Death that cannot be attributed to a CTCAE term associated with Grade 5. 